\title{
RESEARCH ON NON-INVASIVE LASER DOPPLER CURRENT MEASUREMENT FOR HYDROTHERMAL VENTS FLOW RATES PROFILE
}

\author{
Hongwei Zhang ${ }^{1, ~ *, ~ S o n g h u a ~ W u ~}{ }^{1,2}$, Jintao Liu ${ }^{1}$, Yuanshuai Zhang ${ }^{1}$, Xiaomin Chen ${ }^{1}$ \\ ${ }^{1}$ Ocean Remote Sensing Institute, College of Information Science and Engineering, Ocean University of \\ China, Qingdao 266100, China \\ ${ }^{2}$ Laboratory for Regional Oceanography and Numerical Modeling, Pilot National Laboratory for Marine \\ Science and Technology (Qingdao), Qingdao 266237, China \\ *Email: zhanghongwei8944@ouc.edu.cn
}

\begin{abstract}
Hydrothermal vents play important roles in the dynamic process of the stratosphere and the circulation of energy materials. Nowadays, invasive devices such as vane-type flow meter and turbine flow meter are commonly utilized to estimate the flow rates of hydrothermal vents. Insitu observation data obtained by LDCP system and CFD methods will be combined to solve the blind area of LDCP system during in-situ observation measurements. The flow rates profiles obtained in this project will improve the research on the circulation of energy materials and mineralization.
\end{abstract}

\section{INTRODUCTION}

Generally, hydrothermal vents form in volcanically active areas, especially on mid-ocean ridges. Ocean water percolates into the crust through cracks and porous rocks and is heated by underlying magma. The heat helps drive chemical reactions that remove oxygen, magnesium, sulfates and other chemicals from the water that entered the ocean through rain, rivers, and groundwater. In the process, the fluids also become hotter and more acidic, causing them to leach metals such as iron, zinc, copper, lead, and cobalt from the surrounding rocks. Hydrothermal vents act as natural plumbing systems that transport heat and chemicals from the interior of the Earth and that help regulate global ocean chemistry. In the process, they accumulate vast amounts of potentially valuable minerals on the seafloor [1].

A number of different methods have been used to estimate the flow rates of hydrothermal vents since the discovery of vents, starting with visual observations using a vane-type flow meter from the Alvin submersible [2, 3]. Lately, turbine flow meters combined with temperature-probe data allowed the measurement of fluid flow rates exiting from high-temperature vents. The turbine flow meter was held $3 \mathrm{~cm}$ above the fluid exit to limit jamming of the turbine by small particles and to prevent melting of the plastic insulation [4]. However, it was shown that the sensor was significantly affected by fouling during long-term deployment [5]. An alternative method, visual tracking of particles and eddies in the ascending fluids, was used by Rona and Trivett and Ramondenc [6, 7]. In contrast, non-invasive methods that use image processing techniques to track refractive index anomalies can estimate diffuse velocities or matter fluxes [8, 9]. Fluid velocity, however, estimated through video imagery, which requires the presence of eddies and particles and does not allow for autonomous long-term measurements [10].

Nowadays, there are no non-invasive instruments designed for high-resolution flow rates measurements of hydrothermal vents, making the estimation of heat flux with great error [11, 12].

In this paper, in-situ observation data obtained by LDCP system and CFD methods will be combined to solve the blind area of LDCP system during in-situ observation measurements. The detailed information of hydrothermal vents obtained by in-situ devices will be analyzed in this project to confirm the specification of Laser Doppler Current Probe for hydrothermal vent insitu observation. Measurement scheme to in-situ observation of hydrothermal will be designed based on a LDCP system with features of highresolution and non-invasive.

\section{The Laser Doppler Current Profiler system}

The LDCP system is non-invasive flow measurement tool based on LDA (laser Doppler 
anemometry) principles which has been developed to be a high-level standard method for flow measurement. A standard one-component LDA system consists of two laser beams. For simplicity two laser beams of equal frequencies $\left(v_{0}\right)$ are considered to intersect at an angle $2 \alpha$ (Fig. 1). The cross area of these two laser beams in the flow is called the measurement volume. A particle that is suspended in the flow is assumed to pass through the measurement volume and so scatters the lights of two laser beams simultaneously. Because of the different spatial layout of two laser beams, the moving particle of velocity up perceives the different light frequencies resulted from different Doppler effects. A detector is spatially located along $\ell_{2}$ for receiving the light that is scattered from the measurement volume. The velocity of the moving particles are considered to be the speed of flow.

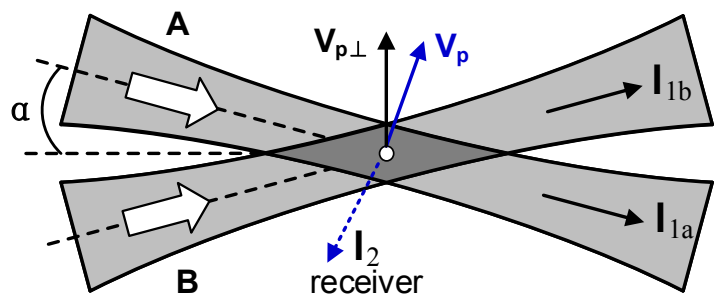

Fig. 1 The scheme of particle passing through the measurement volume formed by LDCP system

A LDCP system based on LDA technique has been designed for the measurements of ocean current as Fig.2 shows. The LDCP system (including: optical transmitting and receiving parts, electronical instruments and data processing devices) is assemabled into a $1 \mathrm{~cm}$ Alumium tube to protect it from the high pressure of sea water.

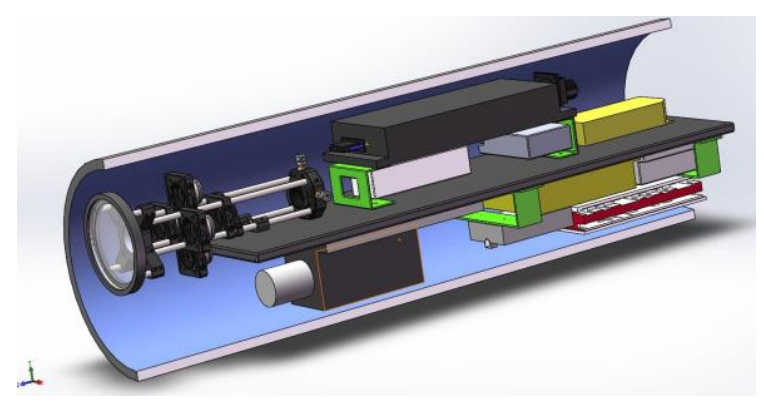

Fig.2 LDCP system assembly diagram generated by Solidworks software

The continuum laser source is a DPSS laser with excellent beam quality (M2=1.1), whose average energy is $50 \mathrm{~mW}$ at each wavelength. The Doppler shift of acoustic optical modulator (AOM) is $40 \mathrm{MHz}$, while the sampling rate of Field Programmable Gate Array (FPGA) is $250 \mathrm{MHz}$ to make the signal accurately. The detailed specifications of the LDCP system are listed in the Table 1.

Table 1 the specification of the LDCP system

\begin{tabular}{cc}
\hline Qualification & Specification \\
\hline Wavelength & $491 \mathrm{~nm} \& 532 \mathrm{~nm}$ \\
Power & $50 \mathrm{mW@each} \mathrm{wavelength}$ \\
Accuracy & $1 \%$ \\
Velocity Range & $0.001-10 \mathrm{~m} / \mathrm{s}$ \\
Measurement Range & $0.2 \mathrm{~m}$ \\
\hline
\end{tabular}

\section{Simulation of Flow Rates}

Computational fluid dynamics (CFD) is a branch of fluid mechanics that uses numerical analysis and data structures to analyze and solve problems that involve fluid flow. Generally, high-speed computers are used to perform the calculations required to simulate the free-stream flow of the fluid, and the interaction of the fluid (liquids and gases) with surfaces defined by boundary conditions.

A model of glass trough has been constructed by Rahino software, and the flow field has been calculated by Fluent software as Fig. 3 shows. The characteristic of the glass trough's central plane is illustrated in Fig. 3. The colors reveal the flow speed while the arrow mean the flow direction.

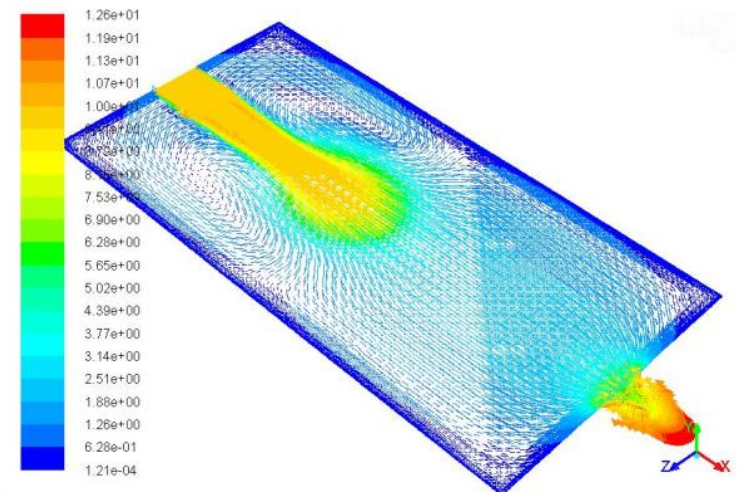

Fig.3 Flow field of glass trough's central plane simulated by Fluent software

To verify the accuracy of simulated results, the flow information (include flow rates and flow 
direction) of selected position in the model can be picked out. The selected positions are illustrated in Fig.4, while the simulated results are listed in Table 2 .

Table 2 simulation of flow rates under different input speed conditions.

\begin{tabular}{lllll}
\hline & $5 \mathrm{~m} / \mathrm{s}$ & $10 \mathrm{~m} / \mathrm{s}$ & $15 \mathrm{~m} / \mathrm{s}$ & $20 \mathrm{~m} / \mathrm{s}$ \\
\hline Point-1 & 1.35 & 5.68 & 11.16 & 5.40 \\
Point-2 & 0.60 & 9.05 & 14.48 & 2.40 \\
Point-3 & 0.97 & 2.07 & 3.63 & 3.88 \\
Point-4 & 0.92 & 1.71 & 2.83 & 3.68 \\
Point-5 & 1.90 & 2.51 & 5.04 & 7.60 \\
Point-6 & 1.14 & 2.40 & 3.65 & 4.56 \\
Point-7 & 1.12 & 1.92 & 2.83 & 4.48 \\
Point-8 & 0.80 & 1.76 & 2.64 & 3.20 \\
Point-9 & 1.87 & 0.74 & 0.95 & 7.50 \\
Point-10 & 5.54 & 10.00 & 15.05 & 22.16 \\
Point-11 & 0.60 & 0.60 & 1.12 & 2.40 \\
\hline
\end{tabular}

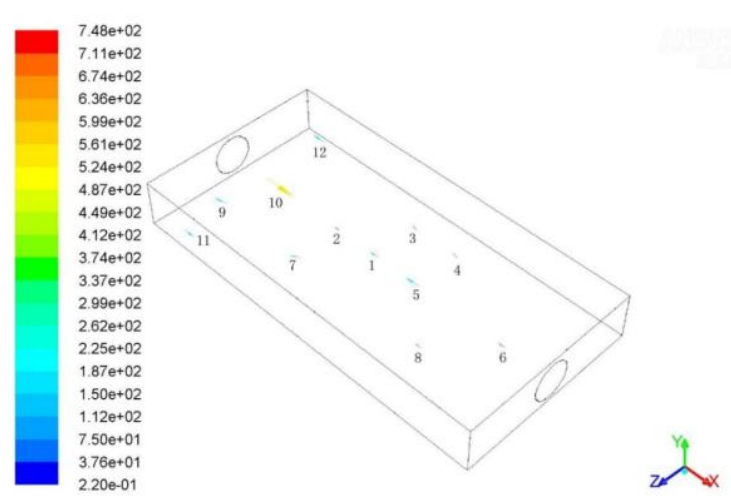

Fig.4 Selected position in flow field simulated by Fluent software

\section{METHODOLOGY}

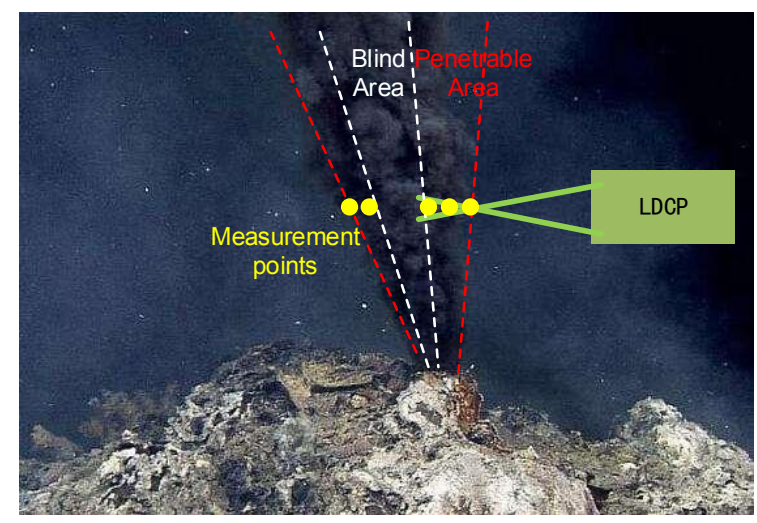

Fig.5 The scheme of hydrothermal vent flow rates profile measurements using the LDCP system

For measurements of flow such as hydrothermal vents flow, non-invasive techniques with high spatial resolution should be applied. Conventional laser Doppler anemometry (LDA) is not capable of obtaining high-spatial-resolution flow rate profiles because of existing of blind area of LDCP system as Fig.5 shows.

To solve the existing of blind area during the insitu measurement, this paper intends to combine the LDA technology and CFD technology to illustrate the whole flow field characteristics of hydrothermal vents. The Schematic diagram of retrieving velocity profile from the fusion of simulated data and measured data can be depicted from Fig.6. The modified laser Doppler current probe was used to measure flow rates in the penetrable area of the hydrothermal vents, and the measured data will be used as the input data of the fluid simulation software to simulate the flow velocity profile of the hydrothermal vents. The input parameters of the simulation software (such as boundary conditions, fluid viscosity, number of iterations, etc.) will be adjusted to fit the measurement curves. Then the whole flow rates profile will be calculated by Fluent software.

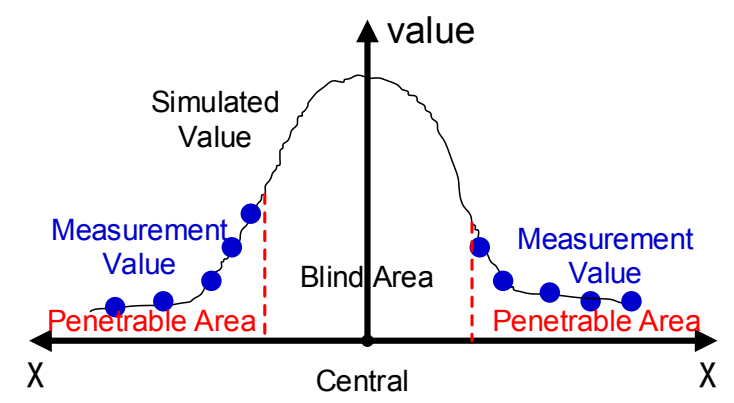

Fig.6 Schematic diagram of retrieving velocity profile from the fusion of simulated data and measured data

\section{CONCLUSION}

For the deep sea in-situ measurements, no technology has enough advantages to replace other measurement techniques, and various measurement methods play a role in the field in which it is good. The traditional devices are invasive instruments with the advantages of low cost and easy operation. Their body shell, however, disturb the flow field and cause large measurement error especially under low speed conditions. Comparing to the point measuring device, the instruments based on the acoustic Doppler technology have large measuring range and high-time resolution. Due to low spatial resolution, the acoustic instruments perform not 
well in high resolution flow rate measurements of hydrothermal vents. Methods based on the optical video image sequences to retrieve the flow information of vertical plane can make full use of the existing seabed hydrothermal video data. The measurement result, however, focus on the surface of the hydrothermal vent and cannot provide the flow rates information inside the hydrothermal spray column.

In this paper, we focus on the combination of LDA technology and CFD technology for flow field measurements based on an optical noninvasive instrument. However, the flow rates profiles vary greatly with different exit speed or the shape of hydrothermal vent models. Table of flow rates under different exit speed or different hydrothermal vent shape conditions will be established to estimate the heat flux of entire hydrothermal vents field.

\section{FUTURE WORK}

The method for measuring the flow rate profiles of the hydrothermal vent models mentioned in this paper needs to be verified by experimental work and simulations. Table of flow rates under different input speed or different hydrothermal vent shape conditions should be constructed to retrieve the detailed information of the flow field.

The temperature and velocity information perform important roles in estimating the heat flux. However, the LDCP system is designed for flow rates measurement. The temperature sensor and conductivity sensor should be combined with the LDCP system during the field experiments for the estimation of heat flux.

Although the method mentioned in this paper can estimate flow rates, measurements encompass small areas, still making estimation of fluid fluxes across an entire vent field difficult without other constraints.

\section{ACKNOWLEDGEMENTS}

We thank our colleagues for their kindly support during the laboratory experiments, including Kailin Zhang and Xiangcheng Chen with Ocean University of China for performing the LCDP system, Lei Liu and Fanghan Wang for CFD simulation work. This research was funded by National Key Research and Development
Program of China under Grant No. 2016YFC1400904.

\section{REFERENCES}

[1] https://www.whoi.edu/main/topic/hydrothermalvents

[2] Corliss, J. B., Dymond, J., et al. Science, 203(4385), 1073-1083 (1979).

[3] Schultz, W., Apicella, P., Scarnati, E., et al. Journal of neuroscience, 12(12), 4595-4610 (1992).

[4] Rona, P. A., et al. Eos, Transactions American Geophysical Union, 83(44), 497-502 (2002).

[5] Smart, C. J., Roman, C., et al. Geochemistry, Geophysics, Geosystems, 14(11), 4743-4757 (2013).

[6] Xu, G., Jackson, D. R., et al. Geochemistry, Geophysics, Geosystems, 14(7), 2369-2382 (2013).

[7] Zhang, X., et al. Deep Sea Research Part I: Oceanographic Research Papers, 57(2), 297-306 (2010). [8] Mittelstaedt, E., et al. Deep Sea Research Part II: Topical Studies in Oceanography, 150, 67-81 (2018).

[9] Dumke, I., Ludvigsen, et al. IEEE Transactions on Geoscience and Remote Sensing (2018).

[10] Sarrazin, J. et al. Deep Sea Research Part I: Oceanographic Research Papers, 56(11), 2065-2074 (2009).

[11] Xu, G., Jackson, D. R., et al. Earth and Planetary Science Letters, 404, 220-231 (2014).

[12] Thomson, R. E., et al. Journal of Geophysical Research: Oceans, 94(C4), 4709-4720 (1989).

[13] Michel, A. P., et al. Deep Sea Research Part II: Topical Studies in Oceanography, 150, 57-66 (2018). 